\title{
Contribuições da enfermagem na prevenção de infecções relacionadas ao cateter venoso central em unidades de terapia intensiva: revisão integrativa
}

\author{
Nursing contributions to the prevention of central venous catheter-related infections in \\ intensive care units: an integrative review
}

Contribuciones de enfermería a la prevención de infecciones relacionadas con el catéter venoso central en unidades de cuidados intensivos: una revisión integradora

Yohanna Cavalcanti de Lima ${ }^{1 *}$, Marta Gleice Firmino ${ }^{1}$, Emmanuela Santos Costa ${ }^{1}$, Thayná Thalita Fabricio Lira Soares ${ }^{1}$, Jéssica Leite Bernardo da Silva ${ }^{1}$, Angélica dos Santos Nobre Ramos ${ }^{1}$, Lara Oliveira Araújo ${ }^{1}$, Elaine Joana Oliveira de Almeida ${ }^{1}$, Rita de Cássia Soares Justino ${ }^{1}$, Leidyanne Soares Gomes².

\section{RESUMO}

Objetivo: Analisar as publicações cientificas sobre as contribuições da equipe de enfermagem na prevenção de infecções relacionadas ao Cateter Venoso Central (CVC) em Unidades De Terapia Intensiva (UTI). Métodos: Trata-se de uma revisão integrativa, realizada a partir da busca nas bases de dados: Banco de Dados em Enfermagem - Bibliografia Brasileira (BDENF), Literatura Latino-Americana e do Caribe em Ciências da Saúde (LILACS) e Sistema Online de Busca e Análise de Literatura Médica (MEDLINE/PUBMED). Através do cruzamento dos descritores: Infecções relacionadas a cateter, Cuidados de Enfermagem e Unidade de Terapia Intensiva, sendo utilizados artigos completos em português, inglês e espanhol, dos últimos 5 anos, após a seleção foram escolhidos 11 artigos para compor está revisão Resultados: Os trabalhos analisados mostraram um conjunto de medidas para prevenção de infecções, que a equipe de enfermagem deve implementar em sua prática para garantir uma assistência segura para o paciente, e as dificuldades enfrentadas por eles para implementa-las. Considerações finais: Os cuidados prestados pela equipe de enfermagem, quando realizados de forma adequada, baseado em evidencias cientificas, diminuiu o risco de aquisição de infecções relacionadas ao uso do CVC nas UTI's, portanto, é preciso investir na capacitação desses profissionais.

Palavras-chave: Infecções relacionadas a cateter, Cuidados de enfermagem, Unidade de terapia intensiva.

\begin{abstract}
Objective: To analyze scientific publications on the contributions of the nursing team in the prevention of infections related to Central Venous Catheters (CVC) in Intensive Care Units (ICU). Methods: This is an integrative review, carried out from the search in the databases: Nursing Database - Brazilian Bibliography (BDENF), Latin American and Caribbean Literature in Health Sciences (LILACS) and Medical Literature Analysis and Retrieval System Online (MEDLINE/PUBMED). By crossing the descriptors: Catheter-Related Infections, Nursing Care and Intensive Care Units, being used complete articles in Portuguese, English and Spanish, from the last 5 years, after selection, 11 articles were chosen to compose this review. Results: The articles analyzed showed a set of measures to prevent infections, that the nursing team must implement in their practice to ensure safe care for the patient, and the difficulties faced by them to implement them. Final considerations: The care provided by the nursing team, when performed properly, based on scientific

${ }^{1}$ Universidade Federal de Pernambuco (UFPE), Recife - PE. *E-mail: yohannacavalcantii@gmail.com

2 Universidade Federal de Pernambuco - Centro Acadêmico de Vitória (UFPE/CAV), Vitória de Santo Antão - PE.
\end{abstract}


evidence, reduced the risk of acquiring infections related to the use of CVC in ICUs, therefore, it is necessary to invest in training these professionals.

Key words: Catheter-related infections, Nursing care, Intensive care units.

\section{RESUMEN}

Objetivo: Analizar publicaciones científicas sobre las contribuciones del equipo de enfermería en la prevención de infecciones relacionadas con el Catéter Venoso Central (CVC) en Unidades de Cuidados Intensivos (UCl). Métodos: Se trata de una revisión integradora, realizada mediante la búsqueda en las bases de datos: Base de Datos en Enfermería - Bibliografía Brasileña (BDENF), Literatura Latinoamericana y del Caribe en Ciencias de la Salud (LILACS) y Sistema Online de Búsqueda y Análisis de Literatura Médica (MEDLINE / PUBMED). Cruzando los descriptores: Infecciones relacionadas con catéter, Unidad de Cuidados de Enfermería y Cuidados Intensivos, utilizando artículos completos en portugués, inglés y español, de los últimos 5 años, luego de la selección, se eligieron 11 artículos para componer esta revisión. Resultados: Los estudios analizados mostró un conjunto de medidas de prevención de infecciones que el personal de enfermería debe implementar en su práctica para garantizar una atención segura al paciente, y las dificultades que enfrentan para implementarlas. Consideraciones finales: La atención brindada por el personal de enfermería, cuando se realizó adecuadamente, con base en la evidencia científica, redujo el riesgo de adquirir infecciones relacionadas con el uso de CVC en $\mathrm{UCl}$, por lo que es necesario invertir en la formación de estos profesionales.

Palabras clave: Infecciones relacionadas con catéteres, Atención de enfermería, Unidades de cuidados intensivos.

\section{INTRODUÇÃO}

Os hospitais são a categoria de serviços de saúde que mais notificam incidentes referentes à assistência de saúde, no ano de 2018, foram 96.113 notificações. Dentre os principais eventos adversos que afetam os usuários nesses serviços, estão as Infecções Relacionadas à Assistência à Saúde (IRAS), que são infecções obtidas após o paciente ser submetido a algum procedimento relacionado a assistência prestada ou uma internação, que venha a estar relacionada a essas ocorrências (BRASIL, 2019; BRASIL, 2021).

As IRAS se constituem como um grave problema de saúde pública e de segurança do paciente, representando um grande impacto sobre o aumento de custos, aumento do tempo de internação e sobre a letalidade nos hospitais. Por conta da grande quantidade de procedimentos invasivos realizados, necessários a manutenção da sobrevida dos pacientes, as Unidades de Terapia Intensiva (UTI) possuem um maior risco para aquisição de infecções (BRASIL, 2017).

Entre as infecções mais comumente associadas à assistência de saúde nas UTI's, estão as Infecções Primárias de Corrente Sanguínea (IPCS), estima-se que, aproximadamente, $60 \%$ dessas infecções estão relacionadas ao uso de Cateter Venoso Central (CVC), se configurando como principal fator de risco. Essa ocorrência está associada a maior frequência na manipulação e a falta de habilidade técnica no uso desse dispositivo e ao seu tempo de permanência mais prolongado (BRASIL, 2017; DASGUPTA S, et al., 2015).

Devido a essa grande incidência das IPCS's relacionadas ao uso de cateter venoso central, foram desenvolvidas estratégias com o objetivo de controlar e prevenir essas infecções, com base em evidências cientificas. As principais medidas de prevenção foram propostas pelo Center for Disease Control and Prevention (CDC), denominadas de Guidelines for the Prevention of Intravascular Catheter-Related Infections, que são um conjunto de intervenções, chamadas de bundles, que precisam ser colocados em prática de forma sistematizada em cada etapa da assistência prestada (BRACHINE JDP, et al., 2012).

Dentre os cuidados que compõem o bundle, estão a higiene das mãos, precauções de barreira máxima (uso de equipamentos de proteção individual: máscara cirúrgica, capote, gorro, luvas estéreis), assepsia da 
pele utilizando clorexidina alcoólica na inserção do cateter, escolha do sítio de inserção, troca de curativos e avaliação diária da necessidade de permanência do CVC e retirada dos que não são mais necessários ou apresentem sinais de infecção (O'GRADY NP, et al., 2011).

O cuidado do paciente internato na UTI, em uso do CVC, é responsabilidade de uma equipe multiprofissional, mas é importante destacar o papel da enfermagem no controle e prevenção das infecções, visto que esses profissionais exercem um cuidado diário e contínuo ao paciente no ambiente hospitalar, sendo responsável pelos cuidados de manutenção até a remoção do CVC. Portanto, é preciso que esses eles estejam capacitados, com conhecimento teórico e treinamento prático para realizar o manejo de forma segura desses dispositivos, a fim de evitar agravos a saúde do paciente (DANTAS GD, et al., 2017).

Desse modo, o presente trabalho teve por objetivo analisar as publicações cientificas que abordem as contribuições da equipe de enfermagem para a prevenção de infecções relacionadas ao uso do cateter venoso central em unidades de terapia intensiva.

\section{MÉTODOS}

Trata-se de uma revisão integrativa da literatura, construída a partir da busca nas bases de dados: Banco de Dados em Enfermagem - Bibliografia Brasileira (BDENF), Literatura Latino-Americana e do Caribe em Ciências da Saúde (LILACS) e Sistema Online de Busca e Análise de Literatura Médica (MEDLINE/PUBMED). Foram utilizados os descritores presentes na relação de Descritores em Ciências da Saúde (DeCS): Infecções Relacionadas a Cateter, Cuidados de Enfermagem e Unidade de Terapia Intensiva, bem como seus correlatos no Medical Subject Headings (MeSH). O cruzamento dos descritores foi feito por meio do operador boleano "and", da seguinte forma: Infecções Relacionadas a Cateter AND Cuidados de Enfermagem AND Unidade de Terapia Intensiva.

A coleta dos artigos utilizados foi realizada no mês de junho de 2021, por dois juízes independentes. A pergunta norteadora de pesquisa do estudo foi: Quais as evidências cientificas acerca das contribuições da equipe de enfermagem na prevenção de infecções relacionadas ao cateter venoso central na Unidade de Terapia Intensiva?

Os critérios de inclusão foram: estudos disponíveis online, com acesso gratuito, texto disponível na integra, nos idiomas inglês, português e espanhol, que foram publicados nos últimos 5 anos (2016-2021), relacionados ao tema proposto e que respondessem questão norteadora. Foram excluídos estudos com metodologia de revisão de literatura, teses, dissertações, resumos de congressos, anais, artigos de opinião, artigos incompletos, artigos duplicados e artigos não disponíveis na íntegra.

\section{RESULTADOS E DISCUSSÃO}

A busca nas bases de dados resultou em 101 artigos encontrados, dos quais foram excluídos 49 artigos por terem sido publicados a mais de 5 anos e selecionados 52 artigos, desses foram escolhidos 35 pela leitura do título, excluindo os artigos não disponíveis na integra e com outras metodologias, foram selecionados 21 pela leitura do resumo, desses foram excluídos 10 por estarem duplicados, sendo 11 lidos na integra e incluídos nesta revisão. A Figura 1 apresenta um fluxograma que demonstra o processo de seleção dos artigos. 
Figura 1 - Fluxograma da seleção dos artigos adaptado do modelo PRISMA.

\section{Identificação de estudos por meio de banco de dados}

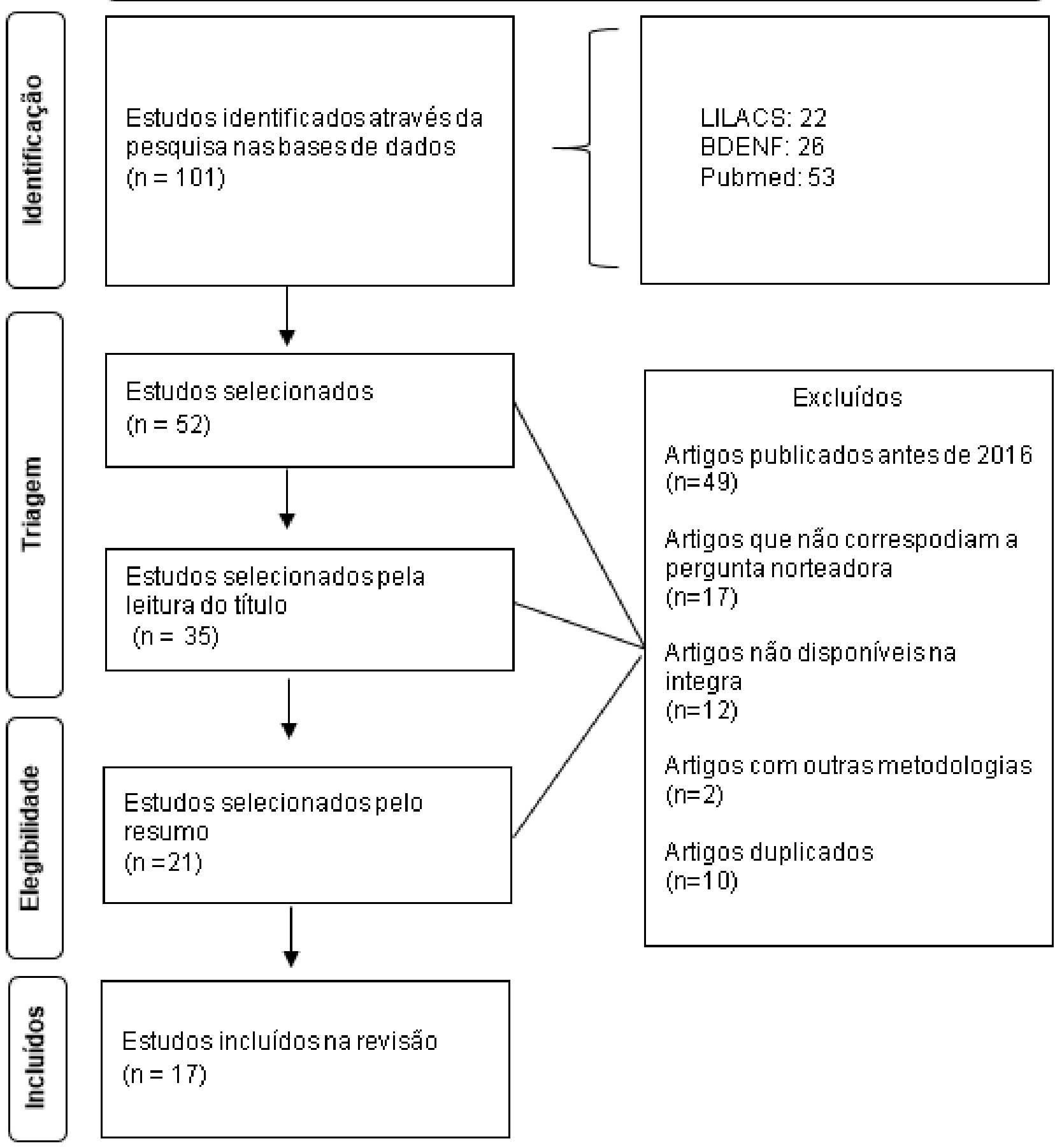

Fonte: LIMA YC, et al., 2021.

Dos artigos publicados nessa revisão, um (9\%) foi publicado no ano de 2021, dois (18\%) no ano de 2020 , um (9\%) publicado em 2019, no ano de 2018 foram publicados 4 artigos (36\%) e em 2017 foram 3 artigos (28\%) publicados. Sendo os anos mais produtivos sobre o tema, os anos de 2018 e 2017. As principais informações sobre os artigos analisados foram organizadas no Quadro 1. 
Quadro 1 - Síntese dos principais achados da pesquisa.

\begin{tabular}{|c|c|c|c|c|c|c|}
\hline $\mathbf{N}$ & $\begin{array}{l}\text { Base de } \\
\text { Dados }\end{array}$ & Título & Autores (Ano) & Objetivo & $\begin{array}{l}\text { Tipo de } \\
\text { Estudo }\end{array}$ & Principais achados \\
\hline 1 & BDENF & $\begin{array}{l}\text { Adesão da equipe de } \\
\text { enfermagem às medidas de } \\
\text { prevenção de infecções de } \\
\text { corrente sanguínea }\end{array}$ & $\begin{array}{l}\text { DANTAS GD, } \\
\text { et al. (2017) }\end{array}$ & $\begin{array}{l}\text { Avaliar o conhecimento e } \\
\text { adesão da equipe de } \\
\text { enfermagem às medidas de } \\
\text { prevenção de infecções de } \\
\text { corrente sanguínea } \\
\text { relacionadas ao cateter venoso } \\
\text { central (ICSR-CVC) em Unidade } \\
\text { de Terapia Intensiva }\end{array}$ & $\begin{array}{l}\text { Estudo } \\
\text { descritivo, } \\
\text { exploratório e } \\
\text { quantitativo }\end{array}$ & $\begin{array}{c}\text { Foi constatado que a equipe de } \\
\text { enfermagem em sua maioria não recebeu } \\
\text { treinamento para inserção e manutenção } \\
\text { de CVC, e a maioria desses profissionais } \\
\text { referiu realizar medidas de manutenção do } \\
\text { CVC, entretanto a execução dessas } \\
\text { medidas não foi visualizada no momento } \\
\text { de observação dos cuidados. }\end{array}$ \\
\hline 2 & $\begin{array}{l}\text { LILACS / } \\
\text { BDENF }\end{array}$ & $\begin{array}{c}\text { Adesão ao bundle de } \\
\text { inserção de cateter venoso } \\
\text { central em unidades } \\
\text { neonatais e pediátricas }\end{array}$ & $\begin{array}{l}\text { ARAÚJO FL, } \\
\text { et al. (2017) }\end{array}$ & $\begin{array}{l}\text { Descrever o comportamento } \\
\text { dos profissionais da equipe de } \\
\text { terapia intensiva neonatal e } \\
\text { pediátrica segundo os itens } \\
\text { propostos no bundle de } \\
\text { inserção de cateter venoso } \\
\text { central (CVC) }\end{array}$ & $\begin{array}{l}\text { Estudo } \\
\text { descritivo, } \\
\text { exploratório e } \\
\text { quantitativo }\end{array}$ & $\begin{array}{l}\text { Este estudo mostrou que quase a } \\
\text { totalidade dos profissionais de saúde em } \\
\text { algum momento durante os procedimentos } \\
\text { observados não cumpriu etapas } \\
\text { importantes do bundle de inserção do CVC. } \\
\text { Destacam-se as técnicas incorretas na } \\
\text { realização da antissepsia cirúrgica e no uso } \\
\text { de clorexidina degermante e alcoólica }\end{array}$ \\
\hline 3 & $\begin{array}{l}\text { LILACS / } \\
\text { BDENF / } \\
\text { MEDLINE }\end{array}$ & $\begin{array}{l}\text { Positive Deviance como } \\
\text { estratégia na prevenção e } \\
\text { controle das infecções de } \\
\text { corrente sanguínea na } \\
\text { terapia intensiva }\end{array}$ & $\begin{array}{l}\text { OLIVEIRA FT, } \\
\text { et al. (2017) }\end{array}$ & $\begin{array}{l}\text { Descrever a aplicação do } \\
\text { Positive Deviance como } \\
\text { estratégia na prevenção e no } \\
\text { controle da infecção de corrente } \\
\text { sanguínea. }\end{array}$ & $\begin{array}{l}\text { Estudo } \\
\text { longitudinal e } \\
\text { prospectivo }\end{array}$ & $\begin{array}{l}\text { O estudo sugere a necessidade de maior } \\
\text { investimento no conhecimento teórico/ } \\
\text { prático sobre prevenção de infecções } \\
\text { relacionadas ao CVC das equipes de } \\
\text { saúde, mais especificamente do } \\
\text { investimento na informação, capacitação e } \\
\text { treinamento. }\end{array}$ \\
\hline 4 & $\begin{array}{l}\text { LILACS / } \\
\text { BDENF }\end{array}$ & $\begin{array}{l}\text { Cateter venoso central para } \\
\text { hemodiálise: incidência de } \\
\text { infecção e fatores de risco }\end{array}$ & $\begin{array}{l}\text { SCHWANKE } \\
\text { AA, et al. } \\
(2018)\end{array}$ & $\begin{array}{l}\text { Mensurar a incidência de } \\
\text { infecção em cateter venoso } \\
\text { central de curta permanência } \\
\text { para hemodiálise e identificar os } \\
\text { fatores de risco associados }\end{array}$ & $\begin{array}{l}\text { Estudo de } \\
\text { Coorte } \\
\text { prospectivo }\end{array}$ & $\begin{array}{c}\text { Esse estudo permitiu identificar que os } \\
\text { fatores de risco associados ao cateter } \\
\text { venoso central para hemodiálise não estão } \\
\text { exclusivamente na prática dos } \\
\text { profissionais, porém isso não impede que } \\
\text { as práticas possam ser avaliadas } \\
\text { regularmente com apoio de medidas } \\
\text { educativas que, junto com a implantação } \\
\text { de protocolos }\end{array}$ \\
\hline
\end{tabular}




\begin{tabular}{|c|c|c|c|c|c|c|}
\hline $\mathbf{N}$ & $\begin{array}{c}\text { Base de } \\
\text { Dados }\end{array}$ & Título & Autores (Ano) & Objetivo & $\begin{array}{l}\text { Tipo de } \\
\text { Estudo }\end{array}$ & Principais achados \\
\hline 5 & $\begin{array}{l}\text { LILACS / } \\
\text { BDENF }\end{array}$ & $\begin{array}{l}\text { Segurança do paciente na } \\
\text { assistência de enfermagem } \\
\text { durante a administração de } \\
\text { medicamentos }\end{array}$ & $\begin{array}{l}\text { OLIVEIRA } \\
\text { JKA, et al. } \\
(2018)\end{array}$ & $\begin{array}{l}\text { Avaliar a conformidade da } \\
\text { prática assistencial da equipe } \\
\text { de enfermagem durante a } \\
\text { administração de medicamentos } \\
\text { por cateter vascular central. }\end{array}$ & $\begin{array}{l}\text { Estudo } \\
\text { descritivo, } \\
\text { prospectivo e } \\
\text { observacional }\end{array}$ & $\begin{array}{c}\text { Foi possível identificar potencialidades e } \\
\text { vulnerabilidades presentes no processo. De } \\
\text { forma geral, em nenhum dos } \\
\text { procedimentos observados o profissional } \\
\text { executou todas as ações necessárias para } \\
\text { a garantia da segurança do paciente, } \\
\text { sendo avaliadas como práticas inseguras } \\
\text { ou sofríveis. }\end{array}$ \\
\hline 6 & $\begin{array}{l}\text { LILACS / } \\
\text { BDENF }\end{array}$ & $\begin{array}{l}\text { Conhecimento autorreferido } \\
\text { das equipes médica e de } \\
\text { enfermagem quanto às } \\
\text { medidas de prevenção de } \\
\text { infecção da corrente } \\
\text { sanguínea }\end{array}$ & $\begin{array}{l}\text { SILVA AG e } \\
\text { OLIVEIRA AC } \\
(2018)\end{array}$ & $\begin{array}{l}\text { Avaliar o conhecimento } \\
\text { autorreferido das equipes } \\
\text { médica e de enfermagem } \\
\text { quanto às medidas de } \\
\text { prevenção de infecção da } \\
\text { corrente sanguínea relacionada } \\
\text { ao cateter venoso central. }\end{array}$ & $\begin{array}{l}\text { Estudo } \\
\text { transversal }\end{array}$ & $\begin{array}{l}\text { Constatou-se um conhecimento limitado às } \\
\text { medidas consideradas padrão ouro na } \\
\text { prevenção da infecção da corrente } \\
\text { sanguínea relacionada ao CVC, } \\
\text { principalmente em relação à equipe de } \\
\text { enfermagem e pelo conhecimento } \\
\text { autorreferido às medidas de desinfecção do } \\
\text { hub; registro dos dias de uso do CVC; } \\
\text { tempo recomendado para troca do curativo, } \\
\text { que foi inferior a 50\% }\end{array}$ \\
\hline 7 & BDENF & $\begin{array}{l}\text { Adesão da enfermagem ao } \\
\text { protocolo de infecção de } \\
\text { corrente sanguínea }\end{array}$ & $\begin{array}{l}\text { CRIVELARO } \\
\text { N, et al. (2018) }\end{array}$ & $\begin{array}{l}\text { Verificar a adesão da equipe de } \\
\text { Enfermagem ao protocolo de } \\
\text { infecção de corrente sanguínea } \\
\text { em pacientes em uso de } \\
\text { cateteres intravasculares. }\end{array}$ & $\begin{array}{l}\text { Estudo } \\
\text { quantitativo, } \\
\text { transversal, } \\
\text { observacional } \\
\text { e descritivo }\end{array}$ & $\begin{array}{l}\text { Constatou-se alta adesão da equipe de } \\
\text { enfermagem ao protocolo de prevenção de } \\
\text { infecção da corrente sanguínea, em } \\
\text { relação ao alto índice de conformidade das } \\
\text { ações preventivas, como identificação, } \\
\text { condições de higiene e fixação do } \\
\text { CVC/AVP, identificação do equipo e } \\
\text { presença de fita colorida no CVC. }\end{array}$ \\
\hline 8 & BDENF & $\begin{array}{c}\text { Bundle para a prevenção } \\
\text { de infecção de corrente } \\
\text { sanguínea }\end{array}$ & $\begin{array}{l}\text { FERNANDES } \\
\text { MS, et al. } \\
\text { (2019) }\end{array}$ & $\begin{array}{l}\text { Verificar o conhecimento dos } \\
\text { profissionais intensivistas sobre } \\
\text { o bundle para a prevenção de } \\
\text { infecção de corrente sanguínea } \\
\text { relacionada ao cateter venoso } \\
\text { central e sobre os cuidados no } \\
\text { manejo deste dispositivo. }\end{array}$ & $\begin{array}{l}\text { Estudo } \\
\text { transversal e } \\
\text { quantitativo }\end{array}$ & $\begin{array}{l}\text { Observou-se que, apesar de a maioria dos } \\
\text { profissionais participantes da pesquisa } \\
\text { afirmar ter conhecimento sobre a existência } \\
\text { do bundle de prevenção de infecção de } \\
\text { corrente sanguínea, um percentual } \\
\text { importante dos profissionais ainda não está } \\
\text { ciente sobre os itens que o compõem e } \\
\text { desconhece os cuidados que devem ser } \\
\text { adotados durante a manutenção. }\end{array}$ \\
\hline
\end{tabular}




\begin{tabular}{|c|c|c|c|c|c|c|}
\hline $\mathbf{N}$ & $\begin{array}{c}\text { Base de } \\
\text { Dados }\end{array}$ & Título & Autores (Ano) & Objetivo & $\begin{array}{l}\text { Tipo de } \\
\text { Estudo }\end{array}$ & Principais achados \\
\hline 9 & $\begin{array}{l}\text { LILACS / } \\
\text { BDENF }\end{array}$ & $\begin{array}{l}\text { Segurança na troca de } \\
\text { equipos e Curativos para } \\
\text { cateter vascular Central: } \\
\text { um estudo observacional }\end{array}$ & $\begin{array}{l}\text { LLAPA- } \\
\text { RODRIGUEZ } \\
\text { EO, et al. } \\
(2020)\end{array}$ & $\begin{array}{l}\text { Avaliar a conformidade da } \\
\text { prática assistencial da equipe } \\
\text { de enfermagem no manuseio do } \\
\text { cateter vascular central na troca } \\
\text { de curativos e de equipos em } \\
\text { unidade de terapia intensiva. }\end{array}$ & $\begin{array}{c}\text { Estudo } \\
\text { descritivo, } \\
\text { prospectivo e } \\
\text { observacional }\end{array}$ & $\begin{array}{c}\text { Foi observado que existem fragilidades } \\
\text { durante a realização dos procedimentos } \\
\text { referentes ao manejo do CVC. Em nenhum } \\
\text { dos procedimentos observados o } \\
\text { profissional executou todas as ações } \\
\text { necessárias para tornar o procedimento } \\
\text { seguro. }\end{array}$ \\
\hline 10 & $\begin{array}{l}\text { LILACS / } \\
\text { BDENF }\end{array}$ & $\begin{array}{l}\text { Bundle de Cateter Venoso } \\
\text { Central: conhecimento e } \\
\text { comportamento de } \\
\text { profissionais em Unidades } \\
\text { de Terapia Intensiva adulto }\end{array}$ & $\begin{array}{l}\text { COSTA CAB, } \\
\text { et al. (2020) }\end{array}$ & $\begin{array}{l}\text { Avaliar o conhecimento e o } \\
\text { comportamento dos } \\
\text { profissionais de UTI no que se } \\
\text { refere às ações recomendadas } \\
\text { em bundles de prevenção de } \\
\text { infecção primária de corrente } \\
\text { sanguínea (IPCS) associada ao } \\
\text { uso do CVC }\end{array}$ & $\begin{array}{l}\text { Estudo } \\
\text { Transversal, } \\
\text { descritivo e } \\
\text { quantitativo }\end{array}$ & $\begin{array}{l}\text { O estudo evidenciou as fragilidades no } \\
\text { conhecimento e comportamento dos } \\
\text { profissionais em relação às ações } \\
\text { preconizadas. A realização de treinamentos } \\
\text { e programas de educação permanente } \\
\text { para todos os profissionais de saúde } \\
\text { engajados na inserção e manutenção do } \\
\text { CVC é imprescindível para prevenir a } \\
\text { infecção de corrente sanguínea. }\end{array}$ \\
\hline 11 & $\begin{array}{l}\text { LILACS / } \\
\text { BDENF }\end{array}$ & $\begin{array}{l}\text { Infecções de corrente } \\
\text { sanguínea relacionada A } \\
\text { cateteres centrais: } \\
\text { entendimento e prática da } \\
\text { Equipe de enfermagem }\end{array}$ & $\begin{array}{l}\text { SILVA MMM, } \\
\text { et al. (2021) }\end{array}$ & $\begin{array}{l}\text { Investigar a prática da equipe } \\
\text { de enfermagem acerca das } \\
\text { medidas de prevenção de } \\
\text { infecções da corrente } \\
\text { sanguínea relacionada ao } \\
\text { cateter venoso central em } \\
\text { unidade de terapia intensiva. }\end{array}$ & $\begin{array}{c}\text { Estudo } \\
\text { descritivo, } \\
\text { qualitativo }\end{array}$ & $\begin{array}{l}\text { O estudou encontrou fragilidades na } \\
\text { compreensão dos profissionais de } \\
\text { enfermagem quanto ao conceito clínico, as } \\
\text { vias fisiopatológicas e às medidas de } \\
\text { prevenção de infecções durante a inserção } \\
\text { e a manutenção de CVC. }\end{array}$ \\
\hline
\end{tabular}

Fonte: Lima YC, et al., 2021. 
A partir da análise dos estudos abordados nessa revisão, é possível entender a relevância para o profissional que atua na UTI, de entender o conceito e as repercussões das IRAS, visto que seus cuidados prestados afetam diretamente a saúde dos pacientes. As IRAS estão relacionadas não só a uma estrutura física inadequada nos serviços de saúde, mas principalmente, a falta de qualificação dos profissionais e o desconhecimento das medidas de controle das mesmas (PADOVEZE MC e FORTALEZA CMCB, 2014).

O ambiente hospitalar possui uma maior predisposição para o aparecimento de infecções, sendo a UTI um dos locais com maior risco para adquirir IRAS, por conta dos diversos procedimentos invasivos que são realizados diariamente. Dentre esses procedimentos, o dispositivo intravascular mais utilizado na UTI é o cateter venoso central, que pode ser inserido tanto em artérias como em veias, central ou perifericamente, sendo utilizado para administração de medicamentos, hemoderivados e fluidos, monitorização hemodinâmica e nutrição parenteral (RICKARD CM, et al, 2015; CRIVELARO N, et al., 2018).

Sendo assim, o uso do CVC é essencial no tratamento dos pacientes, mas existem riscos associados à sua utilização, podendo ser destacado, o risco aquisição da Infecção de Corrente Sanguínea Relacionada a Cateter Venoso Central (ICSR-CVC) como a principal complicação proveniente do uso deste dispositivo. De acordo com Centers for Disease Control and Prevention, o CVC é responsável por $90 \%$ das infecções de corrente sanguínea associadas aos cateteres nas Unidade de Terapia Intensiva (O'GRADY NP, et al., 2011).

A ICSR-CVC é qualificada como Infecção Primária da Corrente Sanguínea (IPCS), que é descrita como uma infecção com consequências sistêmicas graves, sepse ou bacteremia, cujo foco primário não pode ser identificado, quando o CVC se encontra inserido no paciente durante o diagnóstico da infecção. O critério para diagnóstico da infecção é laboratorial, com o resultado positivo da hemocultura, mas também é clínico, por meio de sinais e sintomas, como tremores oligúria, febre, hipotensão, que não podem estar associados a outro sitio de infecção (BRASIL, 2021; BRASIL, 2017).

Estudo realizado por Silva MMM, et al. (2021) mostrou que os profissionais de enfermagem, em sua maioria, não conhecem as definições clínicas das infeções de corrente sanguínea. Para o diagnóstico e consequentemente o tratamento adequado, é preciso saber diferenciar a IPCS, de outros tipos de infecção, como a infecção secundaria de corrente sanguínea, que ocorre quando a infecção é proveniente de outro sítio conhecido. Assim como, diferenciar da infecção local, que pode ser identificada pelos sinais flogisticos externos, como a dor local, o rubor e o aumento da temperatura ao redor da inserção, que precisar ser reconhecida para que a mesma não se torne uma infecção sistêmica (BRASIL, 2013).

Existem três vias de penetração dos microrganismos infecciosos no CVC, a via intralumial, que ocorre principalmente nos cateteres de longa permanência, estando associada a manipulação inadequada, como 0 contato do profissional sem a higienização correta das mãos ou administração de medicações sem a realização da desinfecção dos dispositivos. A via extralumial, quando a contaminação ocorre na inserção do cateter sem a utilização de técnicas adequadas ou pela entrada de patógenos da pele, após a formação de biofilme externamente. Enquanto que a via Hematogênica, ocorre a partir de um foco infeccioso já existente (BRASIL, 2010).

Portanto, as principais vias de entrada de microrganismos patógenos vão estar relacionadas diretamente a assistência à saúde prestada pelos profissionais que atuam nas UTI's. Dentre esses profissionais a equipe de enfermagem é a que possuem maior envolvimento nos cuidados relacionados a manutenção e a retirada do CVC, prestando cuidados diretos constantemente. Portanto, conhecer os conceitos e implicações das IRAS, é essencial para garantia de uma assistência adequada, evitando maiores agravos a saúde do paciente, para tanto é necessário conhecer a maneira correta de realizar o manuseio desses dispositivos para prevenir as infecções (RIBEIRO WA, et al., 2018).

Nesse contexto, surgiu a necessidade de padronização de estratégias das práticas tanto de inserção, manuseio e retirada do CVC, essas medidas, atualmente, são descritas em um guia de diretrizes, o Guidelines for the Prevention of Intravascular Catheter-Related Infections, organizado pelo Centers for Disease Control and Prevention (CDC), esse conjunto de estratégias de baixo custo e de fácil aplicação, é chamada de bundle (OLIVEIRA FJG, et al., 2015). 
Estudos já demonstraram a importância da adoção de medidas de forma padronizada, por meio da implementação de bundles, onde foi vista a diminuição nas taxas de infecção em locais que os mesmos foram aplicados como parte da rotina nos cuidados, destacando assim, que medidas simples podem evitar maiores agravos à saúde do paciente e até a morte, diminuindo tempo de internação e evitando maiores custos para os serviços hospitalares (RIBEIRO GKNA, et al., 2014; SCHWANKE AA, et al., 2018).

No bundle de inserção do CVC são descritas algumas práticas para promover maior segurança para paciente, como: Higienização das mãos antes de realizar o procedimento, uso de barreiras máximas de proteção, que vai do uso adequado de equipamentos de proteção individual e uso de campo estéril, utilização de clorexidina alcoólica para degermação da pele antes da inserção do cateter, e dentre os locais de punção, evitar a veia femoral, por conta da flora bacteriana local, que o torna mais propício ao desenvolvimento de ICS (BRACHINE JDP, et al., 2012).

O profissional de enfermagem não é diretamente responsável pela inserção do CVC, que é realizado pela equipe médica, mas o mesmo possui o papel de garantir que as técnicas corretas sejam seguidas, podendo ser realizado através da aplicação de um checklist no momento da inserção, com base nas diretrizes do bundle. Por isso, o enfermeiro, assim como o médico que irá realizar o procedimento, precisa ter 0 conhecimento sobre essas medidas, a fim de diminuir os riscos para o paciente (OLIVEIRA FJG, et al., 2015; VIANA RAPP, et al., 2014).

Contudo, esse cuidado que deveria ser prestado pela equipe de enfermagem na inserção do cateter não é aplicado na prática, como pôde ser evidenciado pelo estudo realizado com esses profissionais onde os mesmo afirmaram que os seus cuidados são exclusivamente no manuseio e manutenção do cateter, nenhum mencionou a aplicação do checklist de inserção do CVC, junto à equipe médica, o que pode contribuir para 0 maior risco de ocorrência de infecções (SILVA MMM, et al., 2021; COSTA CAB, et al., 2020).

As medidas do bundle de manutenção, incluem: a higienização das mãos antes de qualquer manipulação dos dispositivos, verificar diariamente a necessidade de permanência do CVC, evitar manuseio excessivo do cateter, realizar a limpeza do hub ou dos conectores com álcool 70\%, colocar data no hub ou conectores e no equipo. No estudo de Dantas GD, et al (2017), foi verificado que a maioria dos profissionais da equipe de enfermagem não tinha conhecimento de todas as medidas de manutenção do CVC descritas pelo CDC (BRACHINE JDP, et al., 2012).

A higienização das mãos, antes da realização de qualquer procedimento, é uma prática que deve ser aplicada em qualquer ambiente hospitalar, não somente nas UTI's, nesse setor ela se torna ainda mais essencial, por conta dos patógenos presentes no ambiente e o quadro grave dos pacientes internados. Estudos já mostraram que o contato direto das mãos contaminadas de um profissional com o paciente é uma das principais maneiras de transmitir microorganismos, e apesar de reconhecerem a importância dessa prática, muitos profissionais de enfermagem ainda não incorporaram a sua rotina (OLIVEIRA FJG, et al, 2015; JARDIM JM, et al., 2013).

Em um estudo realizado por Souza LM, et al. (2015), mostrou que em 446 oportunidades que os profissionais tiveram para adesão da prática de higienização das mãos, na UTI, somente $43,7 \%$ aderiram. Corroborando com esses resultados, outros estudos mais recentes realizados com profissionais de enfermagem, também mostraram baixa adesão a essa pratica, além da realização de maneira inadequada da técnica (LLAPA-RODRíGUEZ EO, et al., 2020; ARAÚJO FL, et al., 2017).

Existem algumas dificuldades referidas pelos profissionais da equipe de enfermagem para não aplicação dessa prática, como a escassez de recursos humanos, que provoca uma sobrecarga dos profissionais, resultando em pouco tempo para realização dos procedimentos, o próprio esquecimento, a distância da pia do local de realização do procedimento, a falta de material ou a não distribuição adequado dos dispensadores, são barreiras enfrentadas por esses profissionais (GIORDANE AT, et al., 2014).

Outro importante ponto abordado no bundle, é o uso de clorexidina alcoólica para realizar a desinfecção dos dispositivos do CVC, essa limpeza precisa ser feita sempre que for necessário o manuseio do cateter, na troca de curativo ou administração de alguma solução. Visto que os patógenos podem estar presentes na 
superfície dos dispositivos, podendo ser introduzido durante a administração de medicamentos (OLIVEIRA FJG, et al., 2015; OLIVEIRA FT, et al., 2017).

Sobre a troca de curativos, a ANVISA recomenda que, assim como os equipos, os mesmo deve ser sempre datado, a fim de garantir que a troca seja realizada no tempo adequado. A troca do curativo vai depender do tipo de cobertura utilizada, mas sempre deve ser feita a limpeza ao redor na inserção do CVC com clorexidina alcoólica $0,5 \%$ e/ou a $2 \%$, se a cobertura utilizada for a gaze estéril dever ser trocada a cada 48 horas, ou caso esteja úmida, suja ou solta. Outra opção de cobertura é o filme transparente semipermeável a troca é feita a cada 7 dias ou antes se necessário, sendo esse o mais recomendável, pois além de ser possível observa o local de inserção do cateter, a fim de identificar previamente sinais de infecção, evita-se o manuseio excessivo, diminuindo os riscos para o paciente (BRASIL, 2017).

Estudo recente de Silva AG e Oliveira AC (2018) com profissionais da equipe de enfermagem, demonstrou que $71,2 \%$ dos entrevistados afirmaram usar esses tipos de curativo no CVC. E divergindo dos dados encontrado na literatura sobre o tempo para realização da troca de curativo, outro estudo, identificou que os curativos com gaze estéril estavam sendo trocados diariamente em $83,3 \%$ dos casos e a troca do filme transparente a cada cinco dias, em 58,1\%. Resultados que não condizem com as recomendações do CDC (FERNANDES MS, et al., 2019; OLIVEIRA JKA, et al., 2018).

Contudo, é possível entender a dimensão dos cuidados prestados pela equipe de enfermagem, que é a principal categoria envolvida com o manuseio do CVC, sendo os que possuem maiores chances de atuar na prevenção das infecções em sua rotina. Portando, a enfermagem precisar estar capacitada para executar as práticas de prevenção, possuir o conhecimento necessário, para prestar uma assistência adequada e segura para o paciente, reduzindo o risco de complicações decorrentes de seus cuidados (RIBEIRO WA, et al., 2018).

\section{CONSIDERAÇÕES FINAIS}

De acordo com os estudos analisados, é possível ver a importância da utilização de técnicas padronizadas para prevenção de infecções. Destacando o papel da equipe de enfermagem, envolvida direta e indiretamente nos cuidados com o cateter venoso central, mostrando também a necessidade de educação permanente, com capacitação teórica e treinamento prático sobre a prevenção de infecções. Sendo preciso, a realização de novos estudos, para detectar dificuldades que precisam ser superadas e potencialidades para a melhor aplicação dessas técnicas na prática.

\section{REFERÊNCIAS}

1. ARAÚJO FL, et al. Adherence to central venous catheter insertion bundle in neonatal and pediatric units. Revista da Escola de Enfermagem da USP, 2017; 51: e03269.

2. BRACHINE JDP, et al. Care bundle to reduce central venous catheter-related bloodstream infection: an integrative review. Revista Gaúcha de Enfermagem. 2012, 33(4): 200-10.

3. BRASIL. Agência Nacional de Vigilância Sanitária. Critérios Diagnósticos de Infecção Relacionada à Assistência à Saúde. $2013 . \quad$ Disponível em: https://bvsms.saude.gov.br/bvs/publicacoes/criterios_diagnosticos_infeccoes_assistencia_saude.pdf. Acessado em: 25 de junho de 2021.

4. BRASIL. Agência Nacional de Vigilância Sanitária. Medidas de prevenção de infecção relacionada à assistência à saúde. $2017 . \quad$ Disponível em: http://www.riocomsaude.rj.gov.br/Publico/MostrarArquivo.aspx?C=pCiWUy84\%2BR0\%3D . Acessado em: 25 de junho de 2021.

5. BRASIL. Agência Nacional de Vigilância Sanitária. Programa Nacional de Prevenção e Controle de Infecções Relacionadas à Assistência à Saúde (PNPCIRAS) 2021 a 2025. 2021. Disponível em: https://www.gov.br/anvisa/ptbr/centraisdeconteudo/publicacoes/servicosdesaude/publicacoes/pnpciras_2021_2025.pdf. Acessado em: 25 de junho de 2021.

6. BRASIL. Agência Nacional de Vigilância Sanitária. Infecção de Corrente Sanguínea: Orientações para Prevenção de

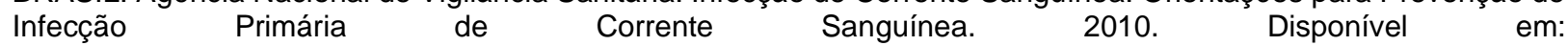
https://www.saudedireta.com.br/docsupload/1340372157manual_orientacao_prevencao_ics_set_2010_anvisa.pdf. Acessado em 25 de junho de 2021. 
7. COSTA CAB, et al. Central Venous Catheter bundle: professional knowledge and behavior in adult Intensive Care Units. Revista da Escola de Enfermagem da USP, 2020; 54: e03629.

8. CRIVELARO N, et al. Adesão da enfermagem ao protocolo de infecção de corrente sanguínea. Revista de Enfermagem da UFPE online, 2018; 12(9): 2361-7.

9. DANTAS GD, et al. Adesão da equipe de enfermagem às medidas de prevenção de infecções de corrente sanguínea. Revista de Enfermagem da UFPE online, 2017; 11(10): 3698-706.

10. DASGUPTA S, et al. Nosocomial infections in the intensive care unit: Incidence, risk factors, outcome and associated pathogens in a public tertiary teaching hospital of Eastern India. Indian Journal of Critical Care Medicine, 2015, 19(1): 14-20.

11. FERNANDES MS, et al. Bundle para a prevenção de infecção de corrente sanguínea. Revista de Enfermagem da UFPE online, 2019; 13(1): 1-8.

12. GIORDANI AT, et al. The nursing team's compliance with hand hygiene: motivational factors. Revista Rene, 2014; 15(4): 559-68.

13. JARDIM JM, et al. Evaluation of practices for the prevention and control of bloodstream infections in a government hospital. Revista da Escola de Enfermagem da USP, 2013; 43(1): 33-45.

14. LLAPA-RODRÍGUEZ EO, et al. Segurança na troca de equipos e curativos para cateter vascular central: um estudo observacional. Texto e Contexto Enfermagem, 2020; 29: e20190107.

15. O'GRADY NP, et al. Guideline for the Prevention of Intravascular Catheter-Related Infections. Clinical Infectious Diseases, 2011, 52(9): e162-e193

16. OLIVEIRA FJG, et al. Use of clinical indicators in the evaluation of prevention and control practices for bloodstream infection. Texto e Contexto Enfermagem, 2015; 24: 1018-26.

17. OLIVEIRA FT, et al. Positive deviance as a strategy to prevent and control bloodstream infections in intensive care. Revista da Escola de Enfermagem da USP, 2017; 51: e03212.

18. OLIVEIRA JKA, et al. Patient safety in nursing care during medication administration. Revista Latino-Americana de Enfermagem, 2018; 26: e3017.

19. PADOVEZE MC, FORTALEZA CMCB. Infecções relacionadas à assistência à saúde: desafios para a saúde pública no Brasil. Revista Saúde Pública, 2014, 48(6): 995-1001.

20. RIBEIRO GKNA, et al. Nursing professionals trained for the labor market in the state of Minas Gerais. REME Revista Mineira de Enfermagem, 2014; 18(1): 15-20

21. RIBEIRO WA, et al. Cateter venoso central na UTI pediátrica: o enfermeiro intensivista na prevenção e controle das infecções hospitalares. Revista Pró-UniverSUS, 2018; 9(2): 47-52.

22. RICKARD CM, et al. Intravascular device administration sets: replacement after standard versus prolonged use in hospitalised patients-a study protocol for a randomised controlled trial (The RSVP Trial). British Medical Journal Open, 2015; 5(2): $1-7$.

23. SCHWANKE AA, et al. Central venous catheter for hemodialysis: incidence of infection and risk factors. Revista Brasileira de Enfermagem, 2018; 71(3): 1115-21.

24. SILVA AG, OLIVEIRA AC. Conhecimento autorreferido das equipes médica e de enfermagem quanto às medidas de prevenção de infecção da corrente sanguínea. Texto e Contexto Enfermagem, 2018; 27(3): e3480017

25. SILVA MMM, et al. Infecções de corrente sanguínea relacionada a cateteres centrais: entendimento e prática da equipe de enfermagem. Revista Online de Pesquisa: Cuidado é Fundamental, 2021; 13: 640-645.

26. SOUZA LM, et al. Adherence to the five moments for hand hygiene among intensive care professionals. Revista Gaúcha de Enfermagem, 2015; 36(4): 21-8

27. VIANA RAPP, et al. Perfil do enfermeiro de terapia intensiva em diferentes regiões do brasil. Texto e Contexto Enfermagem, 2014; 23(1): 151-159. 